\section{BCL-2 double take}

\section{By Lauren Martz, Staff Writer}

Researchers at the Burnham Institute for Medical Research and the Dana-Farber Cancer Institute have independently identified peptides that modulate mitochondrial apoptosis and may have promise in cancer. The therapeutic strategies are, however, quite different: in the first case, the peptides target antiapoptotic B cell CLL/lymphoma 2 family members; in the latter case, the peptides activate proapoptotic B cell CLL/lymphoma 2 proteins.

The Burnham team showed that a short peptide-NuBCP-9-acts as a molecular switch that converts the normally antiapoptotic B cell CLL/ lymphoma 2 (BCL2; BCL-2) into a proapoptotic protein. ${ }^{1}$ Although several companies are developing therapeutics aimed at eliminating BCL-2 and its tumor-protective functions, the newly discovered peptide could be more effective because the proapoptotic conformation actually initiates the elimination of the cancer cells.

The Dana-Farber group showed that targets in the pathway should not be limited to the prosurvival proteins. The team found that activating BCL2-associated X protein (BAX) with stable stapled peptides that bind to a newly identified activation site also could effectively induce apoptosis (see Figure 1, "Switching on apoptosis").

The group has formed a company, Aileron Therapeutics Inc., to develop such activators. ${ }^{2}$

\section{BCL-2 conversion}

BCL-2 is highly expressed in many types of cancer cells and promotes survival by interfering with the mitochondrial programmed cell death pathway., It is a member of a family of six antiapoptotic proteins, including BCL-2 and BCL2-like $1\left(\mathrm{BCL} 2_{\mathrm{L} 1} ; \mathrm{BCL}-\mathrm{X}_{\mathrm{L}}\right)$, and several proapoptotic proteins, such as BAX and BCL2-like 11 (apoptosis facilitator) (BCL2 ${ }_{\mathrm{L} 11}$; BIM), and their activators.

The antiapoptotic members of the family sequester the proapoptotic ones and their activators, forming complexes that neutralize the latter, thus preventing apoptosis. Although this process occurs in all cell types to control cell division and apoptosis, significant upregulation of BCL-2 in cancer cells disrupts the normal balance.

The Burnham team previously showed that nuclear receptor subfamily 4, group A, member 1 (NR4A1; NUR77), a proapoptotic nuclear receptor, bound to BCL-2's large unstructured loop and converted BCL-2 from an antiapoptotic molecule to a proapoptotic molecule in response to certain death signals. ${ }^{5}$

In a new paper published in Cancer Cell, Arnold Satterthwait, Xiao-kun Zhang and colleagues at Burnham identified a short peptide derived from NUR77's BCL-2 binding region that induces a conformational change to BCL-2. Satterthwait is an associate professor in the Infectious Diseases Program at Burnham and Zhang is a professor in the Tumor Development Program.

The nine-amino-acid peptide, dubbed NuBCP-9, induced the conformational change in BCL-2 by binding to the same unstructured loop. The change exposed the protein's BCL2 homology domain 3 (BH3) and allowed activation of the proapoptotic molecules BAX and BCL2-antagonist/killer 1 (BAK1; BAK) as well as the downstream apoptosis that they initiate.

Moreover, the change to BCL-2 prevented the antiapoptotic activity of BCL-X $\mathrm{X}_{\mathrm{L}}$.

NuBCP-9 induced apoptosis in cancer cell lines and suppressed growth of cancer cell xenografts in mice compared with what was seen using a control peptide.

NuBCP-9 also induced more apoptosis in immortalized T lymphocyte Jurkat cells expressing BCL-2 than that seen in wild-type Jurkat cells that do not express BCL-2, and it caused more apoptosis in mouse embryonic fibroblasts (MEFs) than that seen in Bcl-2 knockout MEFs, suggesting that the peptide's activity is dependent on Bcl-2.

\section{Conform to outperform}

Because at least eight companies are already developing therapeutics designed to eliminate BCL-2 activity (see Table 1, "BCL-2 pipeline"), a key question is whether it's better to alter the protein's conformation or eliminate it altogether.

Satterthwait thinks converting BCL-2 into a proapoptotic peptide will be more effective because BCL-2 is not the only antiapoptotic BCL-2 family member overexpressed in cancer cells.

"If you eliminate BCL-2, you may still have to neutralize BCL$\mathrm{X}_{\mathrm{L}}$ or any of the four additional antiapoptotics," Satterthwait said. "Cells keep a lot of backup when it comes to controlling cell death machinery. Thus, neutralizing BCL-2 alone may have no effect on a cell if there are other antiapoptotics around that can neutralize the proapoptotics."

Raymond Warrell, chairman and CEO of Genta Inc., agreed. "Conversion of BCL-2 to an active proapoptotic configuration, rather than reducing amounts of an actively antiapoptotic function that still has residual effects, could be valuable," he said.

Genta is developing Genasense oblimersen, an antisense molecule that targets BCL-2 mRNA to inhibit production of the protein in cancer cells. "Antisense does not convert the antiapoptotic activity of BCL-2; it reduces that effect," Warrell noted.

An NDA for Genasense to treat chronic lymphocytic leukemia (CLL) is under FDA review and has a Dec. 3 PDUFA date. The compound is in Phase III testing for advanced melanoma.

\section{Advancing NuBCP-9}

NuBCP-9 or small molecule mimetics could have utility in a variety of advanced cancers, as well as in early-stage disease.

BCL-2 is overexpressed in cancer cells, and as a result, antagonists are expected to be relatively safe to normal cells. The protein also is associ- 
Figure 1. Switching on apoptosis. The identification of NuBCP-9 as a molecular switch to turn normally antiapoptotic $B$ cell CLL/lymphoma 2 (BCL2; BCL-2) into a proapoptotic protein opens the door to new cancer therapeutics, including mimetics of NuBCP-9.

[a] BCL-2 and BCL2-like 1 (BCL2 ${ }_{L 1}$; $B C L-X_{L}$ ) normally interfere with the mitochondrial programmed cell death pathway by sequestering proapoptotic proteins like BCL2-associated $X$ protein (BAX) and BCL2-antagonist/killer 1 (BAK1; BAK).

[b] But in the presence of NuBCP-9, researchers found that $\mathrm{BCL}-2$ undergoes a conformational change that prevents the protein from binding to BAX and BAK. The conformational change exposes BCL-2's BCL2 homology domain $3(\mathrm{BH} 3)$, which the researchers believe causes the protein to function like BCL-2 family members containing only $\mathrm{BH} 3$ domains.

[c] Under conditions of cellular stress, BCL-2 family members containing only $\mathrm{BH} 3$ domains, including BCL2-like 11 (apoptosis facilitator) (BCL2 ${ }_{\mathrm{L} 11}$; BIM), are activated and bind to $B C L-2$ and $B C L-X_{L}$, preventing $B C L-2$ and $B C L-X_{L}$ from binding to proapoptotic proteins and inhibiting apoptosis.

$\mathrm{BH} 3-$ only proteins also directly activate the proapoptotic proteins BAX and BAK.

[d] Proapoptotics localize to the mitochondria from the cytoplasm to induce membrane permeability.

[e] Cytochrome $\mathrm{C}$ is released from the mitochondria and binds apoptotic peptidase activating factor 1 (APAF1), which forms a complex with caspase-9.

[f] The complex activates additional caspases that lead to cellular death.

ated with resistance to conventional cancer drugs because it functions to protect the cells from apoptosis. Cells with high levels of BCL-2 generally respond less to apoptosis-inducing drugs.

"Cancer cells are loaded with BCL-2," noted Satterthwait. "There are more than 20 types of cancers highly expressing the protein. It was first discovered in leukemia cells and is also found in breast, lung and prostate cancers, to name a few."

George Morris, COO of ValiRx plc, agreed that NuBCP-9 could have efficacy in later-stage disease because high levels of BCL-2 are seen in resistant cancers. He added: "Given that it is relatively benign, with not a lot of toxicity, this could eventually be used for earlier treatment."

ValiRx's VAL 101, an oligonucleotide-protein fusion molecule against BCL-2, is in preclinical development to treat pancreatic cancer. Morris expects VAL 101 to enter clinical testing in the next 12 months.

Some researchers said developing a small molecule mimetic of NuBCP-9 could be preferable to developing the peptide itself.

Jean Viallet, CMO of Gemin X Pharmaceuticals Inc., noted that "the peptide origin of the described compounds are usually limiting in terms of developing the entity as a drug," he said.
Gemin X's obatoclax is a small molecule pan-BCL-2 inhibitor in Phase II testing for follicular and relapsed or refractory Hodgkin's lymphoma.

Morris agreed with Viallet. "Peptides have problems," he said. "By nature, they are immunogenic, and after a few uses they inoculate the person against the molecule." He added that half-lives for peptides are generally on the scale of minutes rather than hours.

Morris also had some concern about the high concentrations of NuBCP-9 that were used in the Cancer Cell paper. Up to $30 \mu \mathrm{M}$ of NuBCP-9 "seem necessary because the peptide has to act with a significant proportion of the BCL-2 expression in the cells. Probably about $70-80 \%$ of all BCL- 2 expression in a given cell would have to be in the new conformation for the therapy to be effective," just like with BCL-2 inhibitors, he said.

Morris argued that a well-designed mimetic could require lower concentrations because its pharmacokinetics and specificity could be improved over the peptide.

Another challenge, said Warrell, is that peptides will readily degrade in vivo, particularly after systemic administration. 
Table 1. BCL-2 pipeline. There are at least seven compounds in development that target B cell CLL/lymphoma 2 (BCL-2). Status is provided for the lead indication.

\begin{tabular}{llll}
\hline Company & Product & Description & Indication \\
\hline Genta Inc. (OTCBB:GNTA) & Genasense oblimersen & $\begin{array}{l}\text { Antisense agent targeting BCL-2 } \\
\text { mRNA }\end{array}$ & $\begin{array}{l}\text { Relapsed chronic lymphocytic } \\
\text { leukemia (CLL) }\end{array}$ \\
\hline Ascenta Therapeutics Inc. & AT-101 gossypol & $\begin{array}{l}\text { Small molecule pan-inhibitor of } \\
\text { BCL-2 family of proteins }\end{array}$ & Various cancers \\
\hline Gemin X Pharmaceuticals Inc. & Obatoclax (GX15-070) & $\begin{array}{l}\text { I.v. formulation of small molecule } \\
\text { pan-inhibitor of BCL-2 proteins }\end{array}$ & Hematological; solid tumor cancers Phase II \\
\hline $\begin{array}{l}\text { Abbott Laboratories (NYSE: } \\
\text { ABT)/Genentech Inc. (NYSE:DNA) }\end{array}$ & ABT-263 & BCL-2 protein antagonist & $\begin{array}{l}\text { CLL; small cell lung cancer; } \\
\text { lymphoid malignancies }\end{array}$ \\
\hline Coronado Biosciences Inc. & CNDO103 apogossypol & BCL-2 inhibitor & Various cancers \\
\hline ProNAi Therapeutics Inc. & PNT2258 & $\begin{array}{l}\text { Cancer therapeutic targeting DNA } \\
\text { sequence upstream of } \\
\text { BCL-2 promoters }\end{array}$ & $\begin{array}{l}\text { Hematologic disease; solid } \\
\text { tumor cancers }\end{array}$ \\
\hline ValiRx plc (LSE:VAL) & VAL 101 & $\begin{array}{l}\text { Oligonucleotide-protein fusion } \\
\text { molecule against BCL-2 }\end{array}$ & Pancreatic cancer
\end{tabular}

Zhang agreed that a mimetic might be preferable. "Small molecules are generally druggable, so we are exploring them along with peptides. They offer better delivery," he said.

However, he and Satterthwait told SciBX they have not ruled out development of a peptide therapeutic because the D-peptide enantiomer of NuBCP-9, which is a more stable configuration of the peptide, is also effective at converting BCL-2.

"One of the most significant findings in the paper is that an enantiomer, a structurally mirrored image, worked as well as the original peptide. We believe that this is because the peptide binds the large, unstructured loop of BCL-2," said Satterthwait.

"Peptides are often ignored as drug candidates since they cannot get into the cells and because they are susceptible to proteolysis. Drug designers favor small molecules since they can be adapted to small fixed binding pockets, are often cell permeable and can't be hydrolyzed. However, using a proteolytically stable $\mathrm{D}$-peptide might overcome those challenges," he said.

\section{BAX to the future}

Meanwhile, Loren Walensky and colleagues at Dana-Farber identified an $\alpha$-helical peptide that activates BAX, and they have characterized the binding site of the peptide on BAX. In a paper in Nature, the researchers showed that, like $\mathrm{NuBCP}-9$, this peptide is able to induce apoptosis in vitro.

Walensky is assistant professor of pediatrics at Harvard Medical School and medical director of the program in cancer chemical biology at Dana-Farber. He is also a consultant and scientific advisor to Aileron.

"Prior to this report, a binding site for triggering BAX was unknown," Walensky told SciBX. "Pharmacologic methods for reactivating the BCL2 family death pathway focused on blocking the survival proteins, but now, we've found a direct way to activate a death protein."
That's good news, he added, because "paradoxically, cancer cells may be especially vulnerable to agents that modulate apoptotic proteins. Normal cells have the ability to withstand stress, while cancer cells are trying to survive in the face of so many stressors that their survival mechanisms can become maxed out. Tipping the balance by targeting proteins in the pathway has the potential to selectively trigger the cancer cells to selfdestruct without reaching that threshold for normal cells."

In order to circumvent some of the problems associated with peptide therapeutics, Walensky's team used its previously published hydrocarbon-stapling technique to enhance the stability of the peptide. ${ }^{6}$ The stapling technique involves stabilizing the peptide's $\alpha$-helical structure using a reaction that generates all-hydrocarbon cross-links.

Walensky said Dana-Farber and Harvard have patented the BAX-activating stapled peptide, and Aileron holds the license to the compound and the stapled-peptide technology.

Tomi Sawyer, SVP and CSO of Aileron, told SciBX the peptide's benefits include its $\alpha$-helical structure, which facilitates interaction with various cellular proteins, and the improved pharmacokinetics resulting from the stapling technique.

"We're seeing optimized peptides with terminal half-lives into the double-digit hours, so we're changing the ability of peptides to function as therapeutics," said Sawyer. He said that before stapling, the company's peptides had half-lives of about five minutes, which is standard for peptides.

Joseph Yanchik, president and CEO of Aileron, added that the technology also allows the molecules to penetrate cells, another frequent problem with peptide therapeutics.

Aileron's most advanced compound is the BAX-activating stapled peptide. Yanchik said the candidate most likely will enter clinical testing within a year to treat leukemia or lymphoma.

Since inception, the company has raised more than $\$ 20$ million, and Yanchik expects the company to raise additional funds in the next year 
to complete preclinical testing and advance the oncology program into the clinic. Investors include Apple Tree Partners and Novartis Venture Funds.

\section{Getting loopy}

With the recent attention the mitochondrial apoptosis pathway is receiving, stapled peptides and NuBCP-9 could be just the beginning. Beyond NuBCP-9, the Burnham researchers expect their finding will open the door for additional therapies targeted to cancer-associated proteins.

"It has recently been realized that large, natively unstructured loops can have considerable functional significance by providing proteins with the adaptability to bind different regulatory partners," said Satterthwait. "Targeting them is likely to play an important role in cancer drug research since more than $50 \%$ of human cancer-associated proteins are predicted to have these large, floppy loops."

Satterthwait and Zhang told SciBX that Burnham has filed a patent application for apoptosis-regulating compounds such as NuBCP-9, and that they are available for licensing.

Martz, L. SciBX 1(39); doi:10.1038/scibx.2008.938

Published online Oct. 30, 2008

\section{REFERENCES}

1. Kolluri, S. et al. Cancer Cell; published online Oct. 6, 2008; doi:10.1016/j.ccr.2008.09.002

Contact: Xiao-kun Zhang, Burnham Institute for Medical Research, La Jolla, Calif.

e-mail: xzhang@burnham.org

Contact: Arnold C. Satterthwait, Burnham Institute for

Medical Research, La Jolla, Calif.

e-mail: asat@burnham.org

2. Gavathiotis, E. et al. Nature; published online Oct. 10, 2008; doi:10.1038/ nature07396

Contact: Loren D. Walensky, Harvard Medical School, Boston, Mass. e-mail: Ioren_walensky@dfci.harvard.edu

Contact: Nico Tjandra, National Institutes of Health, Bethesda, Md. e-mail: tjandran@nhlbi.nih.gov

3. Hockenbery, D. et al. Proc. Natl. Acad. Sci. USA 88, 6961-6965 (1991)

4. Haldar, S. et al. Cancer Res. 58, 1609-1615 (1998)

5. Moll, U. et al. Oncogene 25, 4725-4743 (2006)

6. Walensky, L. et al. Science 305, 1466-1470 (2004)

COMPANIES AND INSTITUTIONS MENTIONED

Aileron Therapeutics Inc., Cambridge, Mass.

Burnham Institute for Medical Research, La Jolla, Calif.

Dana-Farber Cancer Institute, Boston, Mass.

Gemin X Pharmaceuticals Inc., Montreal, Quebec, Canada

Genta Inc. (OTCBB:GNTA), Berkeley Heights, N.J.

Harvard Medical School, Boston, Mass.

ValiRx plc (LSE:VAL), London, U.K. 